\title{
NÍVEL E NATUREZA DO ESTOQUE ORGÂNICO DE LATOSSOLOS SOB DIFERENTES SISTEMAS DE USO E MANEJO ${ }^{1}$
}

\author{
PEDRO LUIZDE FREITAS ${ }^{2}$, PHILIPPE BLANCANEAUX³, EVELYNE GAVINELLI, \\ MARIE-CHRISTINE LARRÉ-LARROUY ${ }^{4}$ e CHRISTIAN FELLER ${ }^{5}$
}

\begin{abstract}
RESUMO - O papel fundamental da matéria orgânica (MO) justifica o crescente interesse pela identificação de sistemas de uso e manejo que melhorem o estoque orgânico em solos tropicais. $\mathrm{O}$ objetivo deste trabalho foi analisar variações quantitativas e qualitativas da $\mathrm{MO}$ e caracterizar compartimentos orgânicos em um Latossolo Vermelho-Escuro argiloso sob vegetação natural antropizada (CER), pastagem de longa duração (PAL), pastagem degradada (PAD), e pousio (PAC), comparados com culturas sob preparo convencional (CCL) e plantio direto (PD). Foi encontrada pouca variação dos estoques orgânicos na camada superficial, explicada pela antropização da vegetação em CER, pela não-exportação dos resíduos em PD e CCL e pela prática de pousio em PAC. Fracionamento granulométrico, considerando os compartimentos resíduos vegetais $(20-2.000 \mu \mathrm{m})$, organo-siltoso $(2-20 \mu \mathrm{m})$ e organo-argiloso $(0-2 \mu \mathrm{m})$, mostrou diferenças na qualidade da MO quando comparadas situações edafoambientais semelhantes. Mesmo com pequenas variações, o compartimento resíduos vegetais foi um indicador da evolução dos estoques orgânicos, permitindo a caracterização da degradação nas pastagens e do efeito do plantio direto, quando comparado ao sistema convencional. PD favoreceu a estocagem de C no compartimento organo-argiloso. Solos estudados diferem de outros solos argilosos tropicais pela mais elevada relação $\mathrm{C} / \mathrm{N}$ encontrada nas frações $0-20 \mu \mathrm{m}$.
\end{abstract}

Termos para indexação: matéria orgânica, fração granulométrica, compartimentos orgânicos, Latossolo Vermelho-Escuro, solos argilosos, pastagens, plantio direto, Cerrados.

\section{NATURE AND LEVEL OF ORGANIC STOCK IN CLAYEY OXISOLS UNDER DIFFERENT LAND USE AND MANAGEMENT SYSTEMS}

\begin{abstract}
The important role of organic matter (OM) justifies the growing interest for the identification of more efficient use and management systems for the improvement of organic stock in tropical oxisols. The objective of this work was to analyze quantitative and qualitative OM stock variations and to characterize organic compartments in a clayey Dark-Red Latosol under situations of natural savanna vegetation (CER), long-term (PAL) and degraded grasslands (PAD), and fallow conditions (PAC). These were compared with crops under conventional plowing (CCL) and zero tillage (PD). Small organic stock variation was found in surface layer of studied soils, explained by human disturbance of vegetation in CER, no residue exportation in PD and CCL, and by fallow practice in PAC. Particle-size fractionating, considering the compartments plant residues $(20-2,000 \mu \mathrm{m})$, silt-organic $(2-20 \mu \mathrm{m})$ and clay-organic $(0-2 \mu \mathrm{m})$, showed differences in OM quality, when comparing similar environmental situations. Even with very weak differences, plant residues compartment was an important indicator of organic stock evolution, allowing the characterization of grassland degradation and zero-till effect, when compared with conventional system. PD has favored C stocking in organic-clayey compartment. Studied soils were distinct from other tropical clayey soils by the high $\mathrm{C} / \mathrm{N}$ relationship found in $0-20 \mu \mathrm{m}$ fraction.
\end{abstract}

Index terms: organic matter, granulometric fraction, organic compartments, Dark-Red Latosol, clayey soils, pasture, zero tillage, acid savannas (Cerrados).

\footnotetext{
${ }^{1}$ Aceito para publicação em 4 de agosto de 1999.

2 Eng. Agrôn., Ph.D., Embrapa-Centro Nacional de Pesquisa de Solos (CNPS), Rua Jardim Botânico, 1024, CEP 22460-000 Rio de Janeiro, RJ. Bolsista do CNPq. E-mail: pfreitas@cnps.embrapa.br
}

\footnotetext{
${ }^{3}$ Pedólogo, Dr., IDR (ex-ORSTOM), 213, Rue La Fayette, 75480 Paris Cedex 10, França.

${ }^{4}$ IDR/LCSC, 911 Av. Agropolis, 34032 Montpellier Cedex 1, França.

${ }^{5}$ Pedólogo, Dr., IDR/LCSC.
} 


\section{INTRODUÇÃO}

Vários trabalhos desenvolvidos nas zonas intertropicais têm demonstrado o importante papel desempenhado pela matéria orgânica (MO) das camadas de superfície, principalmente sobre as principais propriedades edáficas que intervêm na fertilidade do solo (Boyer, 1982; Moreau, 1983; Lepsch et al., 1994; Feller et al., 1996). Tratando-se de pesquisas que procuram formas de redução da emissão de gases que possam estar influenciando o efeito estufa, existe interesse cada vez maior na identificação dos sistemas de manejo de culturas e pastagens susceptíveis à melhoria do estoque orgânico no solo. As pastagens cultivadas aparecem como alternativa com forte potencial de armazenamento de C (Albrecht et al., 1992; Feigl et al., 1995). Para as culturas anuais, grande interesse tem sido consagrado aos sistemas de manejo que utilizam o cultivo mínimo do solo (Beare et al., 1994).

Observa-se, na Região de Cerrados do Brasil, o rápido desenvolvimento da atividade pecuária e a intensificação do plantio de culturas anuais. Os solos da região possuem um elevado potencial para a agricultura mecanizada intensiva, uma vez corrigidas suas deficiências químicas (Goedert, 1987), apresentando, sob vegetação natural, propriedades físicas favoráveis (Blancaneaux et al., 1993). Assim, a entrada em cultivo de solos tropicais acarreta, normalmente, uma forte redução nos estoques orgânicos e degradação do conjunto de propriedades edáficas que são associadas (Cerri et al., 1991; Resck et al., 1991). Isto pode explicar a comum, mas não sistemática, baixa de rendimentos, conduzindo a conseqüências nefastas em termos de conservação do solo e da durabilidade da produção vegetal (Pieri, 1989). Mesmo dispondo de alguns dados sobre os estoques orgânicos dos solos cultivados dos Cerrados, esses são pouco numerosos, necessitando acumular mais informações.

Alguns trabalhos foram desenvolvidos no Brasil sobre modificações induzidas pelo efeito de diferentes sistemas de uso e manejo sobre a dinâmica da MO do solo nas regiões Centro-Sul (Cerri et al., 1985; Feller et al., 1991b) e na Região Amazônica (Desjardins et al., 1994). Contudo, esses são inexistentes para a Região Centro-Oeste.
Os trabalhos que visam identificar os compartimentos orgânicos utilizam os fracionamentos físicos (granulométricos e/ou densimétricos), tanto nas zonas temperadas (Elliott \& Cambardella, 1991; Christensen, 1992) como nas tropicais (Feller, 1993). O fracionamento granulométrico do solo permite separar a MO associada às partículas de areia, silte ou argila, que se distingue fortemente por sua morfologia e origem (Tiessen \& Stewart, 1988; Feller et al., 1991c) e por sua composição e dinâmica (Cerri et al., 1985; Martin et al., 1990; Golchin et al., 1995). Tal procedimento parece bem adaptado à análise do efeito dos sistemas de uso e manejo sobre a evolução qualitativa da MO dos solos no meio tropical (Feller, 1979). Trabalhos recentes mostram o interesse desse tipo de aproximação para a identificação dos compartimentos orgânicos funcionais ao lado dos ciclos biogeoquímicos (Feller, 1993).

O objetivo deste trabalho foi quantificar e caracterizar os diferentes compartimentos orgânicos do solo e o efeito de diferentes sistemas de uso e manejo no nível e natureza do estoque orgânico de Latossolos argilosos da Região de Cerrados.

\section{MATERIAL E MÉTODOS}

O estudo foi conduzido em Latossolo Vermelho-Escuro distrófico textura argilosa, sob relevo pouco ondulado, situados em Goiânia e Senador Canedo, região centro-sul de Goiás, nas estações experimentais da Emater-GO, antiga Emgopa $\left(16^{\circ} 32^{\prime}\right.$ latitude S e $49^{\circ} 08^{\prime}$ longitude W). Trata-se de um solo muito intemperizado em que predominam argilas de baixa atividade e óxidos de ferro e de alumínio, com baixa capacidade de troca catiônica (CTC) e complexo de troca dessaturado. O clima é do tipo subúmido, com duas estações bem definidas, pluviosidade de $1.500 \mathrm{~mm}$ e temperatura média de $23^{\circ} \mathrm{C}$.

As situações consideradas como representativas dos sistemas de uso e manejo do solo na Região de Cerrados, em especial na região central do Estado de Goiás, são: (1) CER - vegetação natural de campo Cerrado (savana arbórea), considerada referência, que apresenta um caráter antrópico característico da região, com desmatamento seletivo, vegetação secundária de gramíneas e introdução de gado durante a estação seca; amostragem realizada em novembro de 1994; (2) PAL - pastagem de longa duração, plantada há mais de 15 anos e reformada em 1989 com aração, fertilização e plantio de Brachiaria 
brizantha; área com pastejo controlado, evitando o superpastoreio (amostragem: agosto de 1994); (3) PAD - pastagem degradada de B. brizantha, plantada há mais de dez anos; superpastoreio e forte infestação de ervas daninhas (novembro de 1993); (4) PAC - pousio por dois anos, com vegetação espontânea de Brachiaria sp. e Penisetum sp., após colheita de cultura de mandioca (Manihot sp.) em área com longo histórico de uso por culturas hortícolas, arroz, soja e milho (novembro de 1993); (5) CCL - cultura de feijão irrigado com preparo convencional de longa duração com grade pesada ou aradora, no máximo a $15 \mathrm{~cm}$ de profundidade, seguida de dupla passagem de grade niveladora; resíduos das culturas anteriores (milho e feijão) incorporados, e controle de ervas daninhas com herbicida não seletivo (trifluralina); amostragem em agosto de 1994, 45 dias após o plantio da cultura de feijão; (6) PD - cultura de milho sob sistema de plantio direto, amostrado em novembro de 1993, 30 dias após plantio sobre resíduos de feijão e aplicação de herbicida dessecante sistêmico (glyphosate).

As parcelas das situações CCL e PD foram instaladas em 1989, após calagem corretiva e homogeneização com uma cultura de feijão, em área com mais de 20 anos sob rotações de culturas hortícolas e arroz.

As áreas foram selecionadas segundo sua representatividade e considerando o histórico de uso e manejo do solo. Em cada área foram realizadas amostragens simples nas profundidades 0-10, 10-20 e 20-40 cm em nove pontos, representando $900 \mathrm{~m}^{2}$. As amostras foram consideradas individualmente e em conjunto (amostras compostas). A densidade do solo (Ds) foi determinada com auxílio de cilindros de $100 \mathrm{~cm}^{3}$ (média de duas repetições).

As determinações analíticas foram realizadas segundo metodologia da Embrapa (1979) nos laboratórios da Embrapa-Centro Nacional de Pesquisa de Solos (CNPS). Os teores de $\mathrm{C}$ e $\mathrm{N}$ totais foram determinados em amostras compostas nos laboratórios do do IDR (ex-ORSTOM) e CIRAD, Montpellier, França. Características químicas e físicas do solo sob as diferentes situações consideradas são apresentadas na Tabela 1 .

O fracionamento granulométrico foi realizado segundo método descrito por Gavinelli et al. (1995), o qual visa a dispersão máxima do solo com a aplicação de ultra-som para as frações inferiores a $50 \mu \mathrm{m}$ e a separação das frações silte e argila pela amostragem de alíquotas. A sequiência de fracionamento granulométrico é a seguinte: 20 g de solo são agitados durante duas horas de maneira rotativa $(50 \mathrm{rpm})$ com $300 \mathrm{~mL}$ de água desionizada em presença de $0,5 \mathrm{~g}$ de hexametafosfato de sódio (HMP) e cinco esferas de vidro. A suspensão é peneirada em água a 200 e a $50 \mu \mathrm{m}$. As frações restantes nas peneiras são lavadas em água, e secadas a $60^{\circ} \mathrm{C}$, pesadas e finalmente trituradas a $20 \mu \mathrm{m}$. A suspensão $0-50 \mu \mathrm{m}$ é submetida a tratamento de ultra-som (10 minutos, $13 \mu \mathrm{m}$ de diâmetro, 600 watts, $90 \%$ de potência, interrupções automáticas de $30 \%$ do tempo total) e peneirada a $20 \mu \mathrm{m}$. A fração 0-20 $\mu \mathrm{m}$ é transferida para proveta de $1000 \mathrm{~mL}$, tendo seu volume completado com água agitada manualmente com 30 rotações. Uma primeira alíquota de $200 \mathrm{~mL}$ é tomada imediatamente com auxílio de pipeta, constituindo a fração 0-20 $\mu \mathrm{m}$. Após repouso de aproximadamente oito horas, é realizada segunda amostragem, constituindo a fração 0-2 $\mu \mathrm{m}$. As duas frações amostradas são isoladas após floculação, com $1 \mathrm{~mL}$ de uma solução saturada de $\mathrm{SrCl}_{2}$, centrifugadas e lavadas no frasco de centrifugação e novamente centrifugadas. A fração floculada é secada à $60^{\circ} \mathrm{C}$, pesada, e triturada a $20 \mu \mathrm{m}$. A fração $2-20 \mu \mathrm{m}$ é calculada por diferença. Os teores de $\mathrm{C}$ e de $\mathrm{N}$ totais do solo não-fracionado (N.F) e das frações separadas foram determinadas com a ajuda de Elementary Autoanalyzer LECO CHN-600 (ORSTOM e CIRAD, Montpellier, França). Os teores de $\mathrm{C}$ e $\mathrm{N}$ totais da fração 2-20 $\mu \mathrm{m}$ foram calculados a partir dos valores obtidos para as frações 0-20 e 0-2 $\mu$ m. Por comparação com o método de separação completa das frações 0-2 e 2-20 $\mu \mathrm{m}$, foi demonstrado que essa sequiência conduz a resultados satisfatórios (Gavinelli et al., 1995). C e N solubilizados durante o fracionamento não foram dosados. Devido à alta repetitividade obtida com o método utilizado, foram realizadas determinações sem repetições.

A fim de avaliar o estado de dispersão obtido por fracionamento granulométrico, uma comparação foi feita com a granulometria do solo obtida por análise mecânica com a destruição completa da MO por repetidos ataques com $\mathrm{H}_{2} \mathrm{O}_{2}(10 \%)$ da $\mathrm{MO}$, aplicando sequência idêntica à descrita.

\section{RESULTADOS E DISCUSSÃO}

Os teores de $\mathrm{C}$ e $\mathrm{N}$ das amostras totais são apresentados na Tabela 2. Coeficientes de variação estão entre 4,2 e 15,2\% (média de 8,4) com relação a $C$, e entre 3,3 e 22,8\% (média de 12,2) com relação a N. Tal variabilidade está de acordo com os valores encontrados em numerosas situações tropicais (Feller,1995). Teores de $\mathrm{C}$ e $\mathrm{N}$ diminuem com a profundidade. Diferenças entre as situações são, geralmente, pequenas nas camadas $0-10$ e $10-20 \mathrm{~cm}$. Valores mais elevados que $10-20 \mathrm{~cm}$, observados em PAC, podem ser explicados pelo 
histórico de manejo, com preparo profundo do solo e incorporação de resíduos, corretivos e adubos, ocorridos até a última cultura de mandioca. Quanto ao conjunto das situações na camada $0-20 \mathrm{~cm}$, os teores de $\mathrm{C}$ variam entre 17 e $22 \mathrm{mg} \mathrm{g}^{-1}$ nas pastagens e nas culturas. Lepsch et al. (1994) citam uma regressão para estimar os teores de $\mathrm{C}\left(\mathrm{em} \mathrm{g} 100 \mathrm{~g}^{-1}\right)$ com base no teor de argila + silte $(\mathrm{A}+\mathrm{S}, 0-50 \mathrm{~mm})$ referente a solos sob vegetação natural e cultivados no Cerrado, que é a seguinte: $\mathrm{C}\left(\mathrm{g} 100 \mathrm{~cm}^{-1}\right)=$ $0,025(\mathrm{~A}+\mathrm{S} \%)+0,628$. Considerando valores

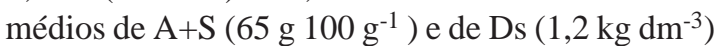
temos um teor de $\mathrm{C}$ estimado de $18,8 \mathrm{mg} \mathrm{g}^{-1}$, o qual está de acordo com aqueles valores obtidos.
As relações $\mathrm{C} / \mathrm{N}$ são elevadas, variando, em todas as amostras, entre 14,1 e 18,2 (Tabela 2). Não existem variações sistemáticas com a profundidade (aumento para CER, CCL e PD, diminuição para PAL e PAC, e aumento e depois diminuição para PAD). Da mesma forma, densidades (Ds) variam entre 1,10 e $1,32 \mathrm{~kg} \mathrm{dm}^{-3}$. A densidade na camada $10-20 \mathrm{~cm}$ é maior ou igual às das camadas superior e inferior, independentemente do sistema de manejo. Isto evidencia a existência de uma camada naturalmente mais adensada, de acordo com as observações morfológicas dos perfis (Blancaneaux et al., 1993; Blancaneaux \& Freitas, 1995). A situação CER apresenta elevada densidade para 0-10 cm, quando

TABELA 1. Características químicas e físicas do solo nas diferentes situações consideradas.

\begin{tabular}{|c|c|c|c|c|c|c|c|c|c|c|c|c|c|c|}
\hline \multirow{2}{*}{$\begin{array}{l}\text { Prof. } \\
(\mathrm{cm})\end{array}$} & \multirow{2}{*}{$\begin{array}{c}\mathrm{PH} \\
\left(\mathrm{H}_{2} \mathrm{O}\right)\end{array}$} & \multirow{2}{*}{$\begin{array}{c}\mathrm{PH} \\
(\mathrm{KCl})\end{array}$} & \multirow[t]{2}{*}{$\mathrm{Al}$} & \multirow[t]{2}{*}{$\mathrm{H}+\mathrm{Al}$} & \multirow[t]{2}{*}{$\mathrm{Ca}+\mathrm{Mg}$} & $\mathrm{K}$ & $\mathrm{S}$ & CTC & \multirow{2}{*}{$\begin{array}{l}\mathrm{V} \\
(\%)\end{array}$} & \multirow{2}{*}{$\begin{array}{c}\mathrm{P} \\
\left(\mathrm{mg} \mathrm{kg}^{-1}\right)\end{array}$} & \multicolumn{2}{|c|}{ Areia } & \multirow[t]{2}{*}{ Silte } & \multirow[t]{2}{*}{ Argila } \\
\hline & & & & & & nol kg-1 & & ----- & & & Grossa & --------------( $\left.\mathrm{g} \mathrm{kg}^{-1}\right)---$ & & \\
\hline \multicolumn{15}{|c|}{ C E R - vegetação natural antropizada de Cerrado } \\
\hline $0-10$ & 5,5 & 4,2 & 0,6 & 6,4 & 0,8 & 0,29 & 1,18 & 7,58 & 16 & $<1$ & 90 & 250 & 200 & 460 \\
\hline $10-20$ & 5,2 & 4,2 & 0,6 & 4,9 & 0,3 & 0,12 & 0,47 & 5,37 & 9 & $<1$ & 90 & 260 & 190 & 460 \\
\hline $20-40$ & 5,4 & 4,3 & 0,4 & 4.0 & 0,3 & 0,09 & 0,43 & 4,43 & 10 & $<1$ & 70 & 250 & 180 & 500 \\
\hline \multicolumn{15}{|c|}{ P A L - pastagem de longa duração não-degradada } \\
\hline $0-10$ & 5,9 & 4,8 & 0,0 & 3,5 & 1,0 & 0,79 & 1,82 & 5,32 & 34 & 2 & 110 & 370 & 160 & 360 \\
\hline $10-20$ & 5,4 & 4,6 & 0,0 & 3,2 & 0,5 & 0,32 & 0,83 & 4,03 & 21 & 1 & 110 & 340 & 170 & 380 \\
\hline $20-40$ & 5,4 & 4,8 & 0,0 & 2,2 & 0,4 & 0,22 & 0,63 & 2,83 & 22 & 1 & 90 & 350 & 150 & 410 \\
\hline \multicolumn{15}{|c|}{ P A D - pastagem de longa duração degradada } \\
\hline $0-10$ & 5,1 & 4,2 & 0,8 & 5,1 & 0,7 & 0,15 & 0,89 & 5,99 & 15 & $<1$ & 110 & 250 & 210 & 430 \\
\hline $10-20$ & 5,1 & 4,2 & 0,6 & 4,3 & 0,4 & 0,08 & 0,51 & 4,81 & 11 & $<1$ & 110 & 260 & 200 & 430 \\
\hline $20-40$ & 5,1 & 4,4 & 0,4 & 3,5 & 0,2 & 0,07 & 0,3 & 3,8 & 8 & $<1$ & 80 & 260 & 170 & 490 \\
\hline \multicolumn{15}{|c|}{ P A C - pousio após cultura } \\
\hline $0-10$ & 5,6 & 4,8 & 0,0 & 4,8 & 2,9 & 0,32 & 3,27 & 8,07 & 41 & 2 & 120 & 200 & 240 & 440 \\
\hline $10-20$ & 5,6 & 4,8 & 0,0 & 4,5 & 2,5 & 0,16 & 2,71 & 7,21 & 38 & 2 & 150 & 230 & 250 & 370 \\
\hline $20-40$ & 5,6 & 4,9 & 0,0 & 4,2 & 1,4 & 0,12 & 1,55 & 5,75 & 27 & 1 & 70 & 200 & 180 & 550 \\
\hline \multicolumn{15}{|c|}{ C C L - cultura com manejo convencional de longa duração } \\
\hline $0-10$ & 5,2 & 4,4 & 0,0 & 5,1 & 1,1 & 0,46 & 1,58 & 6,68 & 24 & 30 & 90 & 260 & 150 & 500 \\
\hline $10-20$ & 4,7 & 4,2 & 0,0 & 4,7 & 0,7 & 0,24 & 0,96 & 5,66 & 17 & 11 & 90 & 250 & 180 & 480 \\
\hline $20-40$ & 4,9 & 4,4 & 0,0 & 3,3 & 0,4 & 0,18 & 0,59 & 3,89 & 15 & 1 & 70 & 270 & 140 & 520 \\
\hline \multicolumn{15}{|c|}{ P D - cultura com plantio direto } \\
\hline $0-10$ & 5,3 & 4,5 & 0,0 & 5,3 & 3,0 & 0,59 & 3,75 & 9,05 & 41 & 43 & 90 & 250 & 150 & 510 \\
\hline $10-20$ & 5,2 & 4,5 & 0,0 & 5,1 & 1,6 & 0,29 & 1,96 & 7,06 & 28 & 13 & 80 & 240 & 170 & 510 \\
\hline $20-40$ & 5,2 & 4,5 & 0,0 & 3,4 & 1,2 & 0,17 & 1,43 & 4,83 & 30 & $<1$ & 70 & 230 & 190 & 510 \\
\hline
\end{tabular}


TABELA 2. Teores e estoques de carbono e nitrogênio totais e relações $\mathrm{C} / \mathrm{N}$ das camadas analisadas.

\begin{tabular}{|c|c|c|c|c|c|c|c|c|}
\hline \multirow{2}{*}{$\begin{array}{l}\text { Prof. } \\
(\mathrm{cm})\end{array}$} & \multicolumn{2}{|c|}{ Carbono } & \multicolumn{2}{|c|}{ Nitrogênio } & \multirow{2}{*}{$\begin{array}{c}\text { Relação } \\
\text { C / N }\end{array}$} & \multirow{2}{*}{$\begin{array}{c}\text { Densidade } \\
\text { do solo } \\
\left(\mathrm{kg} \mathrm{dm}^{-3}\right)\end{array}$} & \multicolumn{2}{|c|}{ Estoque $^{2}$} \\
\hline & $\begin{array}{c}\text { Teor } \\
\left(\mathrm{mg} \mathrm{g}^{-1}\right)\end{array}$ & $\begin{array}{l}\text { C.V. }{ }^{1} \\
(\%)\end{array}$ & $\begin{array}{c}\text { Teor } \\
\left(\mathrm{mg} \mathrm{g}^{-1}\right)\end{array}$ & $\begin{array}{l}\text { C.V. } \\
(\%)\end{array}$ & & & C & N \\
\hline \multicolumn{9}{|c|}{ C E R - vegetação natural antropizada de Cerrado } \\
\hline $0-10$ & 22,6 & 8,8 & 1,53 & 22,8 & 14,8 & 1,19 & 26,9 & 1,82 \\
\hline $10-20$ & 19,6 & 7,4 & 1,22 & 8,2 & 16,1 & 1,24 & 24,3 & 1,51 \\
\hline $20-40$ & 13,7 & 7,8 & 0,82 & 3,3 & 16,7 & 1,12 & 30,7 & 1,84 \\
\hline $0-20$ & & & & & 15,4 & & 51,2 & 3,30 \\
\hline $0-40$ & & 8,0 & & 11,4 & 15,8 & & 81,9 & 5,20 \\
\hline \multicolumn{9}{|c|}{ P A L - pastagem de longa duração não-degradada } \\
\hline $0-10$ & 22,0 & 15,2 & 1,29 & 18,9 & 17,1 & 1,10 & 24,2 & 1,42 \\
\hline $10-20$ & 16,1 & 13,4 & 1,06 & 18,2 & 15,2 & 1,32 & 21,3 & 1,40 \\
\hline $20-40$ & 10,8 & 10,8 & 0,73 & 12,8 & 14,8 & 1,33 & 28,7 & 1,94 \\
\hline $0-20$ & & & & & 16,1 & & 45,5 & 2,80 \\
\hline $0-40$ & & 13,1 & & 16,6 & 15,7 & & 74,2 & 4,80 \\
\hline \multicolumn{9}{|c|}{ P A D - pastagem de longa duração degradada } \\
\hline $0-10$ & 19,0 & 6,4 & 1,35 & 9,7 & 14,1 & 1,10 & 20,9 & 1,49 \\
\hline $10-20$ & 16,4 & 6,3 & 1,07 & 6,1 & 15,3 & 1,23 & 20,2 & 1,32 \\
\hline $20-40$ & 12,2 & 5,1 & 0,85 & 12,3 & 14,4 & 1,23 & 30,0 & 2,09 \\
\hline $0-20$ & & & & & 14,7 & & 41,1 & 2,80 \\
\hline $0-40$ & & 5,9 & & 9,3 & 14,6 & & 71,1 & 4,90 \\
\hline \multicolumn{9}{|c|}{ P A C - pousio após cultura } \\
\hline $0-10$ & 22,5 & 8,6 & 1,40 & 8,0 & 16,1 & 1,05 & 23,6 & 1,47 \\
\hline $10-20$ & 21,1 & 4,9 & 1,35 & 7,1 & 15,6 & 1,22 & 25,7 & 1,65 \\
\hline $20-40$ & 17,1 & 11,7 & 1,13 & 11,6 & 15,1 & 1,16 & 39,7 & 2,62 \\
\hline $0-20$ & & & & & 15,9 & & 49,4 & 3,10 \\
\hline $0-40$ & & 8,4 & & 8,9 & 15,6 & & 89,0 & 5,70 \\
\hline \multicolumn{9}{|c|}{ C C L - cultura com manejo convencional de longa duração } \\
\hline $0-10$ & 21,4 & 11,1 & 1,43 & 13,9 & 15,0 & 1,1 & 23,5 & 1,57 \\
\hline $10-20$ & 19,4 & 7,2 & 1,12 & 13,5 & 17,3 & 1,29 & 25,1 & 1,45 \\
\hline $20-40$ & 13,9 & 10,2 & 0,77 & 13,0 & 18,1 & 1,29 & 35,7 & 1,98 \\
\hline $0-20$ & & & & & 16,1 & & 48,6 & 3,0 \\
\hline $0-40$ & & 9,5 & & 13,5 & 16,8 & & 84,4 & 5,0 \\
\hline \multicolumn{9}{|c|}{ P D - cultura com plantio direto } \\
\hline $0-10$ & 21,7 & 4,2 & 1,35 & 14,1 & 16,1 & 1,15 & 25,0 & 1,56 \\
\hline $10-20$ & 18,1 & 5,7 & 1,09 & 13,6 & 16,6 & 1,36 & 24,6 & 1,48 \\
\hline $20-40$ & 14,2 & 7,1 & 0,78 & 11,5 & 18,2 & 1,16 & 32,9 & 1,81 \\
\hline $0-20$ & & & & & 16,3 & & 49,6 & 3,0 \\
\hline $0-40$ & & 5,7 & & 13,0 & 17,0 & & 82,5 & 4,8 \\
\hline
\end{tabular}


comparada com a camada $20-40 \mathrm{~cm}$, o que pode ser explicado pela ação antrópica e pelo pisoteio de animais (Freitas et al., 1996).

Os estoques de $\mathrm{C}$ e $\mathrm{N}$, considerando a camada de 0-20 cm (Tabela 2), variam da seguinte forma: vegetação natural (CER) culturas (PD e $\mathrm{CCL}) \sim$ pousio $(\mathrm{PAC}) \geq$ pastagens $(\mathrm{PAL}$ e $\mathrm{PAD})$. Na camada 0-40 cm, o sentido da variação é o mesmo, à exceção de PAC, que apresenta valores mais elevados.

Teores $\left(\mathrm{mg} \mathrm{g}^{-1}\right)$ e estoques $\left(\mathrm{t} \mathrm{ha}^{-1}\right)$ obtidos na camada $0-20 \mathrm{~cm}$ (Tabela 2) são comparados com outras situações tropicais, a partir de regressões estabelecidas por Feller (1995) com base em mais de 60 situações da África do Oeste, Antilhas e Brasil em função dos teores de argila e silte fino $(\mathrm{A}+\mathrm{SF}, 0-20 \mathrm{~mm})$, como segue:

a. Vegetação natural ou pousio de longa duração (NCULT - 25 situações):

Teor $=0,49(\mathrm{~A}+\mathrm{SF} \%)-0,23(\mathrm{r}=0,91)$; Estoque $=0,94(\mathrm{~A}+\mathrm{SF} \%)+8,89(\mathrm{r}=0,94)$.

b.Culturas contínuas sem restituição orgânica (CULT - 24 situações):

Teor $=0,294(\mathrm{~A}+\mathrm{SF} \%)+0,32(\mathrm{r}=0,95)$; Estoque $=0,599(\mathrm{~A}+\mathrm{SF} \%)+5,59(\mathrm{r}=0,92)$.

c. Culturas contínuas com restituição orgânica e/ou pousio de duração inferior a cinco anos (CULT+MO - 16 situações):

Teor $=0,376(\mathrm{~A}+\mathrm{SF} \%)+0,02(\mathrm{r}=0,96)$; Estoque $=0,753(\mathrm{~A}+\mathrm{SF} \%)+7,01(\mathrm{r}=0,93)$.

Quanto a A+SF igual a 60\%, estimam-se teores de $\mathrm{C}$ iguais a 29,2, 22,6 e 18,0 $\mathrm{mg} \mathrm{g}^{-1}$ para as situações NCULT, CULT+MO e CULT, respectivamente, os quais são comparáveis aos valores encontrados nas situações sob pastagem e sob culturas (17 a $22 \mathrm{mg} \mathrm{g}^{-1}$ ). Teores de $\mathrm{C}$ sob vegetação natural (21 $\left.\mathrm{mg} \mathrm{g}^{-1}\right)$ aparecem mais fracos que os simulados em NCULT (29,2 $\mathrm{mg} \mathrm{g}^{-1}$ ). Aplicado aos estoques, o cálculo conduz a comparações idênticas, com valores simulados próximos em relação a pastagens e culturas (41 a $50 \mathrm{t} \mathrm{ha}^{-1}$ ) e inferiores quanto a CER (51 t ha-1), este último comparado com $65 \mathrm{t} \mathrm{ha}^{-1}$ estimado para África e Antilhas. Essas diferenças podem ser atribuídas às condições bioclimáticas (clima e nível de restituição orgânica) ou pedológicas (solos com argila 1:1, nitidamente mais ricos em hidróxidos), ou ainda a um estado de maior degradação da vegetação natural antropizada dos Cerrados.

A introdução de culturas ou de pastagens parece exercer pequeno efeito nos teores e estoques de $\mathrm{C}$ nas camadas superficiais $(0-20 \mathrm{~cm})$ (Tabela 2). A única diferença sensível observada concerne a uma diminuição de $20 \%$ do estoque de C sob pastagem degradada (PAD) em relação a CER, cuja diferença de textura existente entre as duas situações conduz a uma superestimativa dessa diferença. A mesma tendência foi observada por Lepsch et al. (1994) quanto a outras situações sob culturas nos Cerrados, mesmo que uma forte diminuição dos teores e estoques de $C$ seja observada em outras situações tropicais após desmatamento e introdução de culturas. Essa ausência de variação pode ser explicada pela antropização da área sob Cerrado (CER), onde o nível de restituição orgânica é inferior àquela com vegetação protegida, e pela sistemática restituição de resíduos ao solo nas situações sob culturas anuais (CCL e PD) e pousio (PAC). Diversos trabalhos mostram que as pastagens cultivadas e bem manejadas podem manter o nível do estoque orgânico nas camadas superficiais, equivalente ao estoque sob vegetação natural correspondente (Albrecht et al., 1992; Desjardins et al., 1994; Correa \& Reichardt, 1995; Feigl et al., 1995). Em Latossolos de Cerrados, Nascimento et al. (1993) indicam efeitos opostos aos geralmente observados, com um aumento dos teores de C, após desmatamento e cultivo.

A relação $\mathrm{C} / \mathrm{N}$ dos solos aparece particularmente elevada (entre 14 e 17) nas camadas 0-10 e 10-20 cm. Nascimento et al. (1993) fornecem, quanto aos Latossolos de Cerrados com valores de $\mathrm{pH}$ ao redor de 5, relações $\mathrm{C} / \mathrm{N}$ entre 10 e 12 no que se refere às situações de Cerrados, culturas e pastagens. Valores médios obtidos por Feller (1995) nos solos com argila 1:1 são compreendidos entre 9 e 15, na África do Oeste, e 11 e 12, nas Antilhas. Valores elevados, referentes à relação $\mathrm{C} / \mathrm{N}$, são uma característica desses solos tropicais, podendo ser atribuídos à forte acidez do solo, que limita a decomposição da MO com elevada relação $\mathrm{C} / \mathrm{N}$. 
Estudo da matéria orgânica associada às frações granulométricas do solo.

Para interpretar os resultados quantitativos (teores de $\mathrm{C}$ e $\mathrm{N}$ ) e qualitativos (relação $\mathrm{C} / \mathrm{N}$ ) das frações granulométricas, apresentados na Tabela 3, dois aspectos metodológicos do fracionamento, o balanço de massa e o estado de dispersão do solo, são discutidos. O balanço de massa das frações (soma das frações recuperadas em relação ao solo não fracionado) varia entre 99 e 106\% (média de 102,5). Essa ligeira superestimativa, caudada pela adsorção do HMP sobre as partículas do solo durante o fracionamento e ligada ao potencial de fixação de fósforo dos Latossolos argilosos, não afeta o balanço de C e N. A fração recuperada de C está entre 90 e 110\% (média de 95,7). Com exceção de CCL $(10-20 \mathrm{~cm})$, as amostras apresentam um déficit que provem essencialmente do carbono hidrosolubilizado das amostras, entre 0 e $4 \%$ (Feller et al., 1991a), não dosado neste trabalho. A fração recuperada de N situa-se entre 81 e $117 \%$ (média de 97,7). A maior variabilidade observada para o $\mathrm{N}$, em relação ao $\mathrm{C}$, deve-se provavelmente à maior susceptibilidade a erros das dosagens. Assim, com algumas exceções (PAD - 0 a $10 \mathrm{~cm}$ para $\mathrm{N}$; CCL - 10 a $20 \mathrm{~cm}$ para $\mathrm{C} \mathrm{e} \mathrm{N}$ ) pode-se considerar os balanços de massa de $\mathrm{C}$ e $\mathrm{N}$ como satisfatórios.

Para melhor visualizar o estado de dispersão obtido pelo fracionamento granulométrico da $\mathrm{MO}$, a repartição das massas das frações foram comparadas às obtidas pela análise mecânica (fracionamento após destruição da MO). As diferenças das repartições para cada uma das frações são apresentadas na Fig. 1 para as amostras obtidas na camada $0-10 \mathrm{~cm}$ de cada situação. Constata-se, em todas as amostras, à exceção de PAD, uma dispersão incompleta das argilas $(0-2 \mu \mathrm{m})$, com diferenças entre 5 e $17 \%$. A fração não dispersa resta, em parte, associada ao silte fino $(2-20 \mu \mathrm{m})$ na forma de microagregados estáveis, normalmente observado nos solos argilosos com forte estabilidade estrutural (Feller, 1995). Nas frações superiores a $20 \mu \mathrm{m}$, observa-se uma boa dispersão, à exceção de CER, na qual a dispersão na fração areia fina (50-200 $\mu \mathrm{m})$ é incompleta. De maneira geral, foi obtida uma boa dispersão dos elementos finos até $20 \mu \mathrm{m}$, com uma dispersão incompleta das argilas que restam, parcialmente associadas ao silte fino $(2-20 \mu \mathrm{m})$.
Considerando-se as características gerais das frações granulométricas em solos com argila 1:1, Feller (1993) propôs reagrupar as diferentes frações granulométricas de MO por sua morfologia, origem, composição e dinâmica. Na fração 20-2.000 $\mu \mathrm{m}$ ou resíduos vegetais a $\mathrm{MO}$ apresenta diversos graus de decomposição, elevadas relações $\mathrm{C} / \mathrm{N}$, entre 15 e 25 , e xylose/mannose, entre 5 e 10 , dependendo da origem vegetal de cada fração, e taxas médias a elevadas de renovação de C em 10 anos (40 a 100\%). $\mathrm{Na}$ fração 2-20 $\mu \mathrm{m}$ ou complexo organo-siltoso a MO é dominada por resíduos vegetais e fúngicos humificados associados a microagregados silto-argilosos muito estáveis, resistentes à dispersão durante fracionamento, e com relações médias de $\mathrm{C} / \mathrm{N}$, entre 10 e 15, e de xylose/mannose, entre 1 e 3 , em função da característica da mistura complexa desta fração, freqüentemente rica em argila, e taxas médias a fracas de renovação de $\mathrm{C}$ em 10 anos (20 a 40\%). A fração 0-2 $\mu$ m ou compartimento organo-argiloso apresenta a MO dominada pelos constituintes orgânicos amorfos e humificados, fortemente ligados aos constituintes argilosos e resíduos bacterianos, fracas relações $\mathrm{C} / \mathrm{N}$, entre 8 e 11 , e xylose/mannose, entre 0,5 e 2 , em função da origem parcialmente microbiana dessa fração, e taxas médias a fracas de renovação de $\mathrm{C}$ em dez anos (20 a 40\%).

Os resultados, agrupados em três frações principais podem ser observados na Tabela 4 . A fração 20-2.000 $\mu \mathrm{m}$ apresenta teores baixos de C, com médias de 7,5 e de 4,4 , nas camadas $0-10$ e $10-20 \mathrm{~cm}$, respectivamente. A contribuição relativa dessa fração é fraca, compreendida entre 13,5 e $24,9 \%$ na camada $0-10 \mathrm{~cm}$ e entre 5,0 e $22,0 \%$ na camada $10-20 \mathrm{~cm}$. Relações C/N têm valores relativamente elevados, variando ligeiramente na camada de $0-10 \mathrm{~cm}$ (14 a 17), sendo mais fracas e mais variáveis na camada de $10-20 \mathrm{~cm}(7$ a 15,5$)$. Na fração 2-20 $\mu$ m ocorrem teores mais altos de $\mathrm{C}$, fortemente dependentes da massa da fração. A contribuição relativa dessa fração é muito variável, entre 22,1 e $46,5 \%$ na camada de $0-10 \mathrm{~cm}$, e entre 28,7 e 43,2\% na camada $10-20 \mathrm{~cm}$. As relações C/N têm valores mais elevados, variando fortemente nas duas camadas. A fração 0-2 $\mu \mathrm{m}$ tem teores médios de $\mathrm{C}$ e contribuição relativa elevada, entre 37,2 e 55,3\% (mé- 
TABELA 3. Características das frações granulométricas da matéria orgânica do solo nas profundidades de 0-10 e $10-20 \mathrm{~cm}$.

\begin{tabular}{|c|c|c|c|c|c|c|}
\hline \multirow[t]{2}{*}{ Fração $(\mu \mathrm{m})$} & \multicolumn{2}{|c|}{ Massa das frações $\left(\mathrm{g} \mathrm{kg}^{-1}\right)$} & \multicolumn{2}{|c|}{ Teor de $\mathrm{C}\left(\mathrm{mg} \mathrm{g}^{-1}\right)$} & \multicolumn{2}{|c|}{ Teor de $\mathrm{N}\left(\mathrm{mg} \mathrm{g}^{-1}\right)$} \\
\hline & $0-10$ & $10-20$ & $0-10$ & $10-20$ & $0-10$ & $10-20$ \\
\hline \multicolumn{7}{|c|}{ C E R - vegetação natural antropizada de Cerrado } \\
\hline $200-2000$ & 81,1 & 48,2 & 1,7 & 0,7 & 0,08 & 0,03 \\
\hline $50-200$ & 387,7 & 303,2 & 2,9 & 0,5 & 0,20 & 0,05 \\
\hline $20-50$ & 63,3 & 70,5 & 0,7 & 0,3 & 0,03 & 0,02 \\
\hline $2-20$ & 77,5 & 156,3 & 4,7 & 7,3 & 0,17 & 0,31 \\
\hline $0-2$ & 393,8 & 445,2 & 11,3 & 10,8 & 0,87 & 0,77 \\
\hline Fração recuperada & $100,3 \%$ & $102,3 \%$ & $94,1 \%$ & $100,1 \%$ & $88,0 \%$ & $97,2 \%$ \\
\hline \multicolumn{7}{|c|}{ P A L - pastagem de longa duração não-degradada } \\
\hline $200-2000$ & 68,0 & 61,7 & 1,5 & 0,5 & 0,06 & 0,02 \\
\hline $50-200$ & 380,5 & 374,3 & 1,2 & 0,6 & 0,11 & 0,06 \\
\hline $20-50$ & 80,6 & 78,1 & 0,5 & 0,2 & 0,00 & 0,0 \\
\hline $2-20$ & 122,8 & 98,2 & 6,0 & 4,4 & 0,34 & 0,23 \\
\hline $0-2$ & 359,8 & 387,8 & 10,6 & 9,5 & 0,80 & 0,7 \\
\hline Fração recuperada & $101,2 \%$ & $100,0 \%$ & $90,2 \%$ & $94,2 \%$ & $106,9 \%$ & $97,9 \%$ \\
\hline \multicolumn{7}{|c|}{ P A D - pastagem de longa duração degradada } \\
\hline $200-2000$ & 71,6 & 70,4 & 0,8 & 0,3 & 0,05 & 0,02 \\
\hline $50-200$ & 320,3 & 321,6 & 1,2 & 0,4 & 0,09 & 0,08 \\
\hline $20-50$ & 76,4 & 459,4 & 0,4 & 0,8 & 0,00 & 0,11 \\
\hline $2-20$ & 144,7 & 211,0 & 5,6 & 6,5 & 0,22 & 0,33 \\
\hline $0-2$ & 439,5 & 385,7 & 9,9 & 8,5 & 0,70 & 0,6 \\
\hline Fração recuperada & $105,2 \%$ & $105,6 \%$ & $94,5 \%$ & $96,4 \%$ & $81.0 \%$ & $97,7 \%$ \\
\hline \multicolumn{7}{|c|}{ P A C - pousio após cultura } \\
\hline $200-2000$ & 41,4 & 47,1 & 0,7 & 0,7 & 0,04 & 0,04 \\
\hline $50-200$ & 278,6 & 326,0 & 2,1 & 3,0 & 0,13 & 0,2 \\
\hline $20-50$ & 62,8 & 66,7 & 0,7 & 0,7 & 0,00 & 0,0 \\
\hline $2-20$ & 314,8 & 289,1 & 10,0 & 8,8 & 0,58 & 0,48 \\
\hline $0-2$ & 323,8 & 309,8 & 8,0 & 7,3 & 0,60 & 0,5 \\
\hline Fração recuperada & $102,2 \%$ & $103,9 \%$ & $95,5 \%$ & $96,8 \%$ & $98,2 \%$ & $95,0 \%$ \\
\hline \multicolumn{7}{|c|}{ C C L - cultura com manejo convencional de longa duração } \\
\hline $200-2000$ & 48,1 & 43,7 & 1,2 & 0,4 & 0,04 & 0,02 \\
\hline $50-200$ & 316,1 & 302,7 & 0,9 & 0,7 & 0,10 & 0,09 \\
\hline $20-50$ & 76,7 & 73,2 & 0,6 & 0,3 & 0,00 & 0,0 \\
\hline $2-20$ & 223,4 & 200,6 & 8,2 & 9,2 & 0,54 & 0,39 \\
\hline $0-2$ & 362,8 & 377,2 & 8,8 & 10,7 & 0,70 & 0,8 \\
\hline Fração recuperada & $102,7 \%$ & $99,7 \%$ & $92,2 \%$ & $109,7 \%$ & $99,0 \%$ & $117,2 \%$ \\
\hline \multicolumn{7}{|c|}{ P D - cultura com plantio direto } \\
\hline $200-2000$ & 483,5 & 438,8 & 1,2 & 0,5 & 0,07 & 0,01 \\
\hline $50-200$ & $3.317,5$ & $3.296,7$ & 1,8 & 1,1 & 0,12 & 0,1 \\
\hline $20-50$ & 620,8 & 616,2 & 0,5 & 0,4 & 0,00 & 0,0 \\
\hline $2-20$ & 236,9 & 204,7 & 8,2 & 6,7 & 0,43 & 0,35 \\
\hline $0-2$ & 322,3 & 365,7 & 8,1 & 8,1 & 0,60 & 0,6 \\
\hline Fração recuperada & $100,1 \%$ & $100,6 \%$ & $91,7 \%$ & $92,6 \%$ & $95,8 \%$ & $98,5 \%$ \\
\hline
\end{tabular}




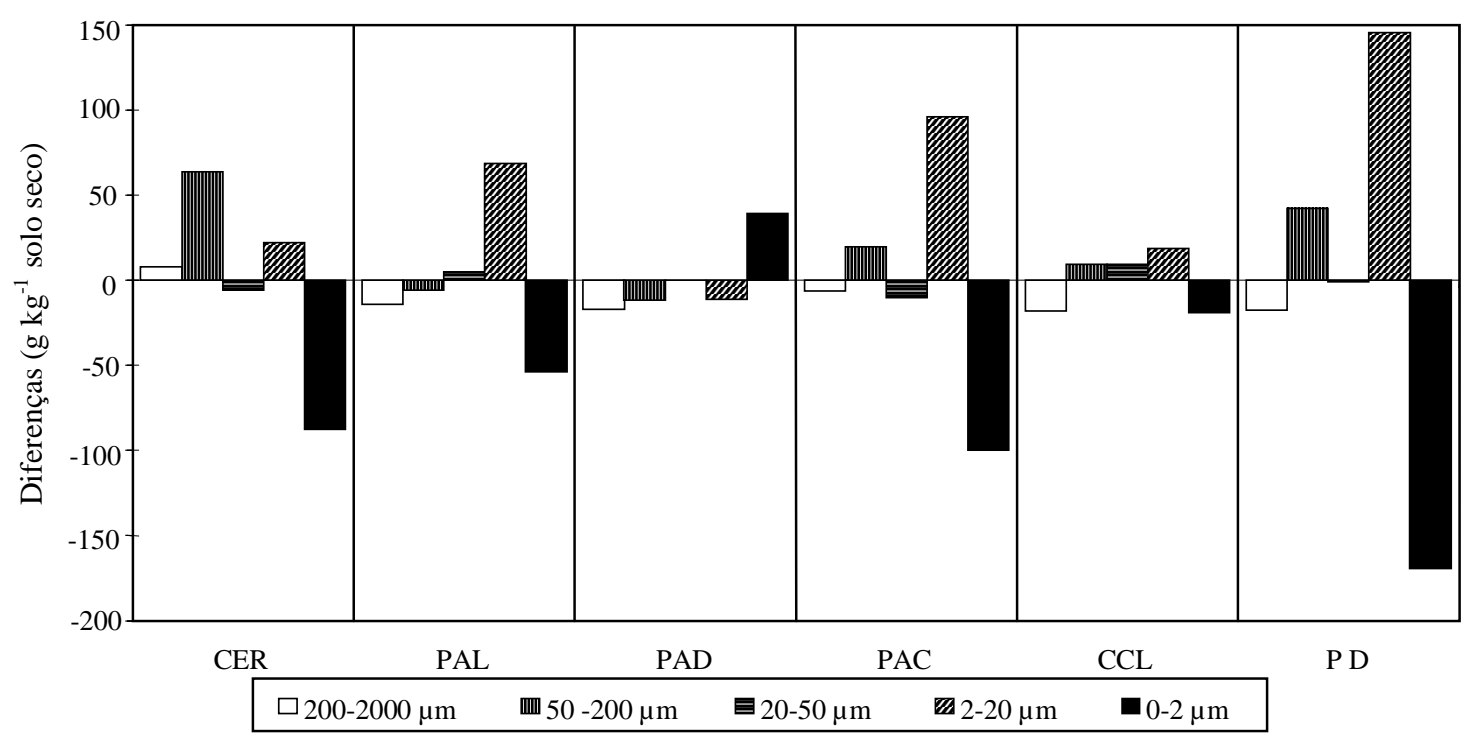

FIG. 1. Diferenças obtidas para as massas das diferentes frações granulométricas, em $\mathrm{g} \mathrm{kg}^{-1} \mathrm{solo} \mathrm{seco,} \mathrm{com} \mathrm{e}$ sem destruição da MO. Camada de 0-10 cm.

TABELA 4. Teores de carbono e nitrogênio nas principais frações, nas profundidades de 0-10 e 10-20 cm.

\begin{tabular}{|c|c|c|c|c|c|c|}
\hline \multirow[t]{2}{*}{$\begin{array}{l}\text { Fração } \\
(\mu \mathrm{m})\end{array}$} & \multicolumn{2}{|c|}{$\begin{array}{c}\text { Teor de C } \\
\left(\mathrm{mg} \mathrm{g}^{-1} \text { fração) }\right.\end{array}$} & \multicolumn{2}{|c|}{$\begin{array}{c}\text { Teor de } \mathrm{N} \\
\left(\mathrm{mg} \mathrm{g}^{-1} \text { fração) }\right.\end{array}$} & \multicolumn{2}{|c|}{$\begin{array}{c}\text { Relação } \\
\text { C/N }\end{array}$} \\
\hline & $0-10$ & $10-20$ & $0-10$ & $10-20$ & $0-10$ & $10-20$ \\
\hline \multicolumn{7}{|c|}{ C E R - vegetação natural antropizada de Cerrado } \\
\hline $20-2000$ & 10,0 & 3,6 & 0,58 & 0,24 & 17,2 & 14,9 \\
\hline $2-20$ & 60,9 & 47,9 & 2,16 & 2,05 & 28,2 & 23,4 \\
\hline $0-2$ & 28,7 & 24,9 & 2,22 & 1,78 & 12,9 & 14,0 \\
\hline \multicolumn{7}{|c|}{ P A L - pastagem de longa duração não-degradada } \\
\hline $20-2000$ & 6,1 & 2,6 & 0,38 & 0,19 & 15,9 & 13,8 \\
\hline $2-20$ & 49,8 & 44,3 & 2,76 & 2,33 & 18,0 & 19,0 \\
\hline $0-2$ & 29,8 & 24,5 & 2,37 & 1,84 & 12,6 & 13,3 \\
\hline \multicolumn{7}{|c|}{ P A D - pastagem de longa duração degradada } \\
\hline $20-2000$ & 5,4 & 1,8 & 0,39 & 0,26 & 14,1 & 6,9 \\
\hline $2-20$ & 40,8 & 32,6 & 1,63 & 1,67 & 25,0 & 19,6 \\
\hline $0-2$ & 23,8 & 23,3 & 1,67 & 1,64 & 14,3 & 14,2 \\
\hline \multicolumn{7}{|c|}{ P A C - pousio após cultura } \\
\hline $20-2000$ & 9,4 & 10,5 & 0,57 & 0,67 & 16,6 & 15,5 \\
\hline $2-20$ & 32,6 & 31,7 & 1,87 & 1,72 & 17,4 & 18,4 \\
\hline $0-2$ & 25,3 & 24,4 & 1,85 & 1,74 & 13,7 & 14,0 \\
\hline \multicolumn{7}{|c|}{ C C L - cultura com manejo convencional de longa duração } \\
\hline $20-2000$ & 6,2 & 3,4 & 0,43 & 0,33 & 14,6 & 10,3 \\
\hline $2-20$ & 37,9 & 45,8 & 2,48 & 1,95 & 15,2 & 23,4 \\
\hline $0-2$ & 25,0 & 28,2 & 1,96 & 2,07 & 12,8 & 13,6 \\
\hline \multicolumn{7}{|c|}{ P D - cultura com plantio direto } \\
\hline $20-2000$ & 8,0 & 4,5 & 0,49 & 0,30 & 16,3 & 14,8 \\
\hline $2-20$ & 34,7 & 32,7 & 1,83 & 1,73 & 18,9 & 18,9 \\
\hline $0-2$ & 25,3 & 22,4 & 2,00 & 1,62 & 12,7 & 13,8 \\
\hline
\end{tabular}


dia de 47,4\%) na camada $0-10 \mathrm{~cm}$ e entre 35,5 e $63,6 \%$ (média de $51,0 \%$ ) na de $10-20 \mathrm{~cm}$. As relações $\mathrm{C} / \mathrm{N}$ e suas variações são as mais fracas das três frações, tanto na camada de $0-10 \mathrm{~cm}(12,6$ a 14,3) como na de $10-20 \mathrm{~cm}$ $(13,3$ a 14,3$)$.

Comparando os resultados ( $\mathrm{mg} \mathrm{g}^{-1}$ fração, \%C total e relação $\mathrm{C} / \mathrm{N}$ ) com os obtidos em outros solos tropicais argilosos por Feller (1995) e Kouakoua (1997), apresentados na Tabela 5, observa-se: Fração 20-2.000 $\mu \mathrm{m}$ - Os resultados são similares aos das situações do Brasil (SP e GO); diferem claramente dos resultados das situações Antilhas e Congo, e apresentam teores de $\mathrm{C}$ e relações $\mathrm{C} / \mathrm{N}$ mais elevadas; Fração 2-20 $\mu \mathrm{m}$ - A diferença mais forte refere-se à relação $\mathrm{C} / \mathrm{N}$, que é particularmente elevada nas situações do Brasil-GO (entre 15 e 28), e bem diferente daquela da situação das frações similares, não somente nos outros solos argilosos, mas também de um grande número de outros solos tropicais (Feller, 1993). Um estudo microscópico, ótico e eletrônico seria necessário para precisar a natureza da MO desta fração, que representa a média de $36 \%$ do $\mathrm{C}$ total. Essa fração contribui fortemente para as relações $\mathrm{C} / \mathrm{N}$ elevadas nesses solos; Fração 0-2 $\mu \mathrm{m}$ - A diferença mais forte refere-se à relação $\mathrm{C} / \mathrm{N}$, que é nitidamente mais elevada na situação Brasil-GO (12,6 a 14,3) do que nas outras situações $(\mathrm{C} / \mathrm{N}<12)$.

Uma das características específicas da MO dos Latossolos Vermelho-Escuros da Região de Cerrados é sua relação $\mathrm{C} / \mathrm{N}$ elevada, devido principalmente à relação das frações 2-20 e 0-2 $\mu \mathrm{m}$. Constata-se que modificações relativamente fracas das características do meio, como as encontradas nas situações do Brasil-SP e Brasil-GO, podem originar fortes variações qualitativas da MO desses solos.

Verificou-se o efeito do sistema de uso e manejo nos diversos compartimentos granulométricos com base no teor de $\mathrm{C}$, na relação $\mathrm{C} / \mathrm{N}$ e no estoque de $\mathrm{C}$ determinados sob vegetação natural e pastagens e sob culturas (Tabela 6).

Nos sistemas sob vegetação natural e pastagens (CER, PAL, PAD) temos, para a fração 20-2.000 $\mu \mathrm{m}$, um teor em C mais elevado em CER, ligeiramente superestimado em conseqüência da dispersão incompleta. Sob pastagens, o teor de C é

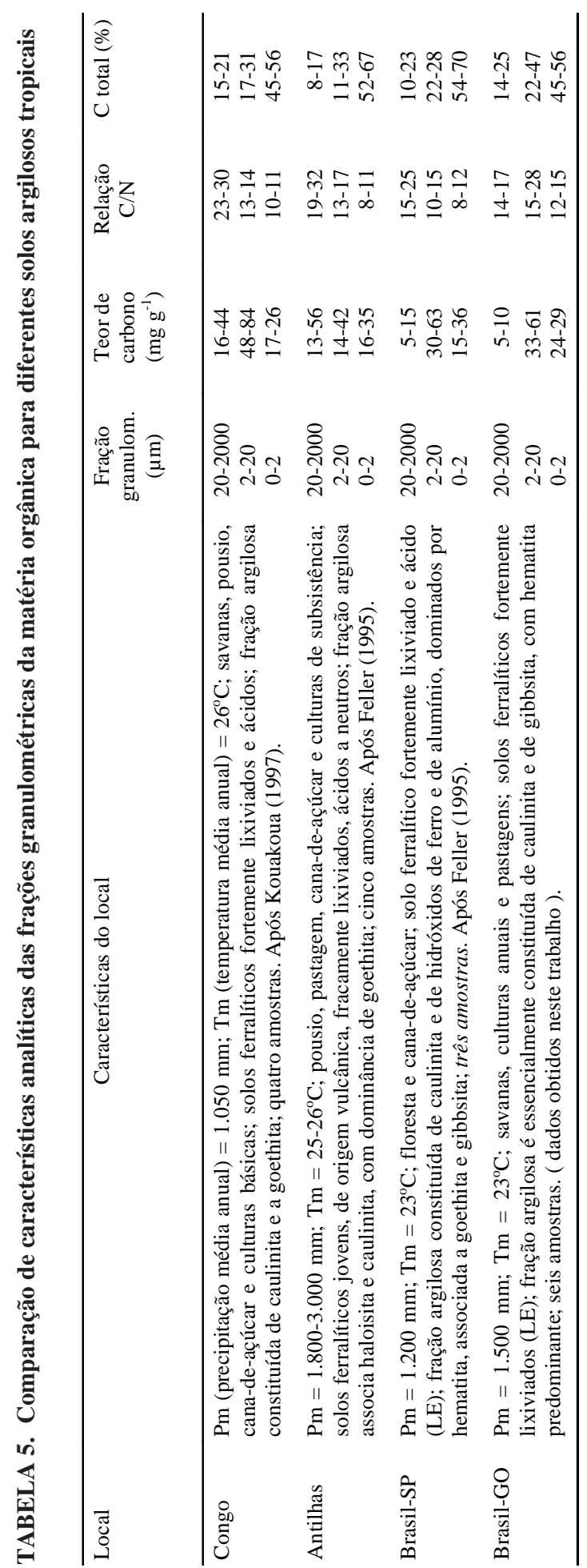


mais elevado em PAL, sendo isto ligado a um nível de produção vegetal mais fraco na situação degradada (PAD), porém com relações $\mathrm{C} / \mathrm{N}$ próximas, o que indica uma qualidade equivalente das restituiç̧ões orgânicas. Os menores valores da relação $\mathrm{C} / \mathrm{N}$ sob pastagem, em relação à situação CER, podem ser atribuídos às restituições mais ricas em $\mathrm{N}$, devido à dejeção dos animais na pasta- gem. As relações $\mathrm{C} / \mathrm{N}$ diminuem com a profundidade, exprimindo a taxa de decomposição mais elevada dessa fração. Na fração 2-20 $\mu$ m, os teores de C são variáveis, sem que haja uma explicação clara. Como assinalado, os valores da relação $\mathrm{C} / \mathrm{N}$ são globalmente mais elevados. Na fração 0-2 $\mu \mathrm{m}$, os teores em $\mathrm{C}$ e relações $\mathrm{C} / \mathrm{N}$ variam pouco com o sistema de uso nas duas camadas.

TABELA 6. Teores de $\mathrm{C}$, relação $\mathrm{C} / \mathrm{N}$ e estoque de $\mathrm{C}$ das diferentes frações granulométricas para sistemas sob vegetação natural, pastagens e culturas.

\begin{tabular}{|c|c|c|c|c|c|c|c|c|}
\hline \multirow[t]{2}{*}{ Variável } & \multirow[t]{2}{*}{$\begin{array}{l}\text { Profund. } \\
(\mathrm{cm})\end{array}$} & \multirow[t]{2}{*}{$\begin{array}{l}\text { Fração } \\
(\mu \mathrm{m})\end{array}$} & \multicolumn{3}{|c|}{$\begin{array}{l}\text { Vegetação natural e } \\
\text { pastagens }\end{array}$} & \multicolumn{3}{|c|}{ Culturas } \\
\hline & & & CER & PAL & PAD & PAC & $\mathrm{CCL}$ & PD \\
\hline \multirow{6}{*}{$\begin{array}{l}\text { Teor de C } \\
\left(\mathrm{mg} \mathrm{g}^{-1} \mathrm{de}\right. \\
\text { solo seco })\end{array}$} & $0-10$ & $20-2000^{1}$ & 5,3 & 3,2 & 2,4 & 3,5 & 2,7 & 3,5 \\
\hline & & $2-20^{2}$ & 4,7 & 6,0 & 5,6 & 10,0 & 8,2 & 8,2 \\
\hline & & $0-2^{3}$ & 11,3 & 10,6 & 9,9 & 8,0 & 8,8 & 8,1 \\
\hline & $10-20$ & $20-2000$ & 1,5 & 1,3 & 0,8 & 4,4 & 1,4 & 1,9 \\
\hline & & $2-20$ & 7,3 & 4,4 & 6,5 & 8,8 & 9,2 & 6,7 \\
\hline & & $0-2$ & 10,8 & 9,5 & 8,5 & 7,3 & 10,7 & 8,1 \\
\hline \multirow[t]{6}{*}{ Relação C/N } & $0-10$ & $20-2000$ & 17 & 16 & 14 & 17 & 15 & 16 \\
\hline & & $2-20$ & 28 & 18 & 25 & 17 & 15 & 19 \\
\hline & & $0-2$ & 13 & 13 & 14 & 14 & 13 & 13 \\
\hline & $10-20$ & $20-2000$ & 15 & 14 & 7 & 16 & 10 & 15 \\
\hline & & $2-20$ & 23 & 19 & 20 & 18 & 23 & 19 \\
\hline & & $0-2$ & 14 & 13 & 14 & 14 & 14 & 14 \\
\hline \multirow{15}{*}{$\begin{array}{c}\text { Estoque } \\
\text { orgânico } \\
\left(\mathrm{t} \mathrm{ha}^{-1}\right)\end{array}$} & $0-10$ & $20-2000$ & 6,3 & 3,5 & 2,7 & 3,7 & 2,9 & 4,1 \\
\hline & & $2-20$ & 5,6 & 6,7 & 6,2 & 10,5 & 9,1 & 9,5 \\
\hline & & $0-2$ & 13,4 & 11,7 & 10,9 & 8,4 & 9,7 & 9,4 \\
\hline & & $0-20^{4}$ & 19,0 & 18,4 & 17,1 & 18,9 & 18,8 & 18,9 \\
\hline & Total & & 25,3 & 21,9 & 19,8 & 22,6 & 21,7 & 23,0 \\
\hline & $10-20$ & $20-2000$ & 1,8 & 1,7 & 1,0 & 5,4 & 1,8 & 2,7 \\
\hline & & $2-20$ & 9,1 & 5,7 & 8,0 & 10,7 & 11,9 & 9,1 \\
\hline & & $0-2$ & 13,4 & 12,5 & 10,5 & 8,9 & 13,8 & 11,1 \\
\hline & & $0-20$ & 22,5 & 18,2 & 18,5 & 19,6 & 25,7 & 20,2 \\
\hline & Total & & 24,3 & 19,9 & 19,5 & 25,0 & 27,5 & 22,9 \\
\hline & $0-20$ & $20-2000$ & 8,1 & 5,2 & 3,7 & 9,1 & 4,7 & 6,8 \\
\hline & & $2-20$ & 14,7 & 12,4 & 14,2 & 21,2 & 21,0 & 18,6 \\
\hline & & $0-2$ & 26,8 & 24,2 & 21,4 & 17,3 & 23,5 & 20,5 \\
\hline & & $0-20$ & 41,5 & 36,6 & 35,6 & 38,5 & 44,5 & 39,1 \\
\hline & Total & & 49,6 & 41,8 & 39,3 & 47,6 & 49,2 & 45,9 \\
\hline
\end{tabular}


O estoque orgânico de $\mathrm{C}$ ( $\left.\mathrm{em} \mathrm{tha}^{-1}\right)$ de cada fração foi analisado na camada de $0-20 \mathrm{~cm}$ (Tabela 6). A situação sob vegetação natural antropizada (CER) apresenta os maiores estoques, em todas as frações, em especial no que diz respeito à fração 20-2.000 $\mu \mathrm{m}$. A pastagem degradada (PAD), comparada com PAL, apresenta uma diminuição sensível do estoque de C na fração 20-2.000 $\mu \mathrm{m}$ e menor, na fração 0-2 $\mu \mathrm{m}$. A tendência inverte-se na fração 2-20 $\mu \mathrm{m}$, devido principalmente aos estoques encontrados na camada de $10-20 \mathrm{~cm}$. A dispersão incompleta das argilas parcialmente associadas ao silte fino causa superestimativa dos estoques de C associados ao complexo organo-siltoso $(2-20 \mu \mathrm{m})$. Por outro lado, sendo a dispersão aceitável para as frações > $20 \mu \mathrm{m}$, pode-se calcular os estoques de C referentes ao conjunto das frações 0-20 $\mu \mathrm{m}$. Os estoques são superiores na situação CER $\left(49,6\right.$ t ha $\left.^{-1}\right)$. Nas condições de cálculo, praticamente não se percebe efeito da degradação da pastagem sobre o estoque de $\mathrm{C}$, associado às frações $<20 \mu \mathrm{m}$.

Comparando-se os três sistemas sob cultura (PAC, CCL e PD), observa-se, na fração 20-200 $\mu \mathrm{m}$, um teor em $\mathrm{C}$ menor na CCL, nas duas camadas (0-10 e 10-20 cm, Tabela 6). Ao contrário, considerando-se que o nível de restituição é equivalente, o sistema de plantio direto (PD) exerce efeito favorável sobre a estocagem de MO nesta fração. A relação $\mathrm{C} / \mathrm{N}$, mais elevada em PAC na camada de $10-20 \mathrm{~cm}$, permite supor taxa de decomposição mais baixa do material orgânico restituído.

$\mathrm{O}$ efeito do pousio (PAC) sobre o aumento dos teores de C na camada de 10-20 cm é clara, considerando-se que a amostragem foi realizada com dois anos de pousio, com densa cobertura de gramíneas. Quanto à fração 2-20 $\mu \mathrm{m}$, os teores em $\mathrm{C}$ variam muito pouco na camada $0-10 \mathrm{~cm}$, e são mais fracos no PD a 10-20 cm. Na fração 0-2 $\mu \mathrm{m}$, os teores de C variam muito pouco na camada $0-10 \mathrm{~cm}$, e as relações $\mathrm{C} / \mathrm{N}$ estão ao redor de 14 , em todas as situações.

Quanto ao estoque de C na camada $0-20 \mathrm{~cm}$, observam-se níveis semelhantes nos três sistemas, situando-se em torno de $7 \mathrm{t} \mathrm{ha}^{-1}$ na fração 20-2.000 $\mu \mathrm{m}$, e em torno de $20 \mathrm{t} \mathrm{ha}^{-1}$, nas demais frações. Comparando com o sistema convencional (CCL), nota-se aumento do estoque de $\mathrm{C}$ de PAC e de PD na fração 20-2.000 $\mu$ m. Os estoques são sensivelmente menores na fração 2-20 $\mu \mathrm{m}$ em PD, e estoques elevados no sistema convencional na fração 0-2 $\mu \mathrm{m}$, devido essencialmente à camada $10-20 \mathrm{~cm}$. Quanto à soma das frações finas $(<20 \mu \mathrm{m})$ na camada $0-20 \mathrm{~cm}$, o sistema convencional CCL permitiu a estocagem de $\mathrm{C}$ associada à frações finas, pelo menos idêntica ou superior às situações com plantio direto (PD) ou o pousio (PAC).

\section{CONCLUSÕES}

1. Os Latossolos argilosos da Região de Cerrados apresentam pouca variação dos estoques orgânicos da camada de 0-20 cm nas situações de uso e manejo consideradas.

2. O fracionamento granulométrico da MO, considerando três compartimentos distintos, permite precisar o efeito de diferentes sistemas de uso e manejo em solos de características edafoambientais semelhantes.

3. Os Latossolos argilosos da Região de Cerrados diferem nitidamente de outros solos argilosos tropicais pela sua relação $\mathrm{C} / \mathrm{N}$ mais elevada nas frações 0-20 $\mu \mathrm{m}$ e principalmente na $0-2 \mu \mathrm{m}$.

4. A degradação das pastagens é caracterizada por um estoque menor de $\mathrm{C}$ do compartimento resíduos vegetais $(20-2.000 \mu \mathrm{m})$ na camada $0-10 \mathrm{~cm}$.

5. O sistema de plantio direto (PD) possibilita maior estocagem de $\mathrm{C}$ no compartimento resíduos vegetais, em comparação com o sistema convencional (CCL).

6. A incorporação dos resíduos no sistema convencional (CCL) favorece a estocagem de C no compartimento organo-argiloso $(0-2 \mu \mathrm{m})$.

7. A fração resíduos vegetais aparece como um indicador da evolução dos estoques orgânicos nos solos, mesmo que estas variações sejam muito fracas.

\section{AGRADECIMENTOS}

Aos pesquisadores e técnicos que participaram nas determinações analíticas: Wilson S. Araújo, Washington de O. Barreto, Marie E.C.C de Melo, José L. de Paula e Daniel V. Peres (Embrapa-CNPS, 
Rio de Janeiro, RJ); Hélio A. de Menezes (Emater-GO, Senador Canedo, GO); Dalmiron P. de Oliveira (Eng. Agrônomo, Bolsista UFG/EA, Goiânia, GO); J. Fardoux e J.C. Marcourel (LCSC/IDR, Montpellier, França).

\section{REFERÊNCIAS}

ALBRECHT, A.; BROSSARD, M.; CHOTTE, J.-L.; FELLER, C. Les stocks organiques des principaux sols cultivés de la Martinique (Petites Antilles). Cahier ORSTOM: Série Pédologie, Paris, v.27, n.1, p.23-36, 1992.

BEARE, M.H.; CABRERA, M.L.; HENDRIX, P.F.; COLEMAN, D.C. Aggregate-protected and unprotected pools of organic matter in conventional and no-tillage soils. Soil Science Society of America. Journal, v.58, p.787-795, 1994.

BLANCANEAUX, Ph.; FREITAS, P.L. de. Utilisation de l'analyse morphostructurale dans les recherches agropédologiques développées dans les Cerrados du Brésil central. Un exemple d'application: Le semis direct sur la paille comme système cultural alternatif vi able. In: RÉ UNION DU GROUPE THÉMATIQUE STRUCTURE ET FERTILITÉ DES SOLS TROPICAUX, 2., 1994, Montpellier. Compte Rendu. Montpellier : ORSTOM, 1995. p.49-56.

BLANCANEAUX, Ph.; FREITAS, P.L. de; AMABILE, R.F.; CARVALHO, A.M. de. Le semis direct comme pratique de conservation des sols des Cerrados du Brésil Central. Cahier ORSTOM: Série Pédologie, Paris, v.28, n.2, p.245-267, 1993.

BOYER, J. Les sols ferrallitiques: Facteur de fertilité et utilisation des sols. Paris : Orstom, 1982. 384p. (ORSTOM. Initiations documentations techniques, $52)$.

CERRI, C.C.; FELLER, C.; BALESDENT, J.; VICTORIA, R.; PLENECASSAGNE, A. Application du traçage isotopique naturel en ${ }^{13} \mathrm{C}$ à l'étude de la dynamique de la matière organique dans les sols. Compte Rendu Académie des Sciences, Paris, tomo 300, Série II, n.9, p.423-428, 1985

CERRI, C.C.; FELLER, C.; CHAUVEL, A. Evolução das principais propriedades de um Latossolo VermelhoEscuro após desmatamento e cultivo por doze e cinqüenta anos com cana-de-açúcar. Cahier ORSTOM: Série Pédologie, Paris, v.26, n.1, p.37-50, 1991.
CHRISTENSEN, B.T. Physical fractionation of soil and organic matter in primary particle size and density separates. Advances in Soil Science, v.20, p.1-19, 1992

CORREA, J.C.; REICHARDT, K. Efeito do tempo de uso das pastagens sobre as propriedades de um Latossolo Amarelo da Amazônia Central. Pesquisa Agropecuária Brasileira, Brasília, v.30, n.1, p.107-114, jan. 1995.

DESJARDINS, T.; ANDREUX, F.; VOLKOFF, B.; CERRI, C.C. Organic carbon and ${ }^{13} \mathrm{C}$ contents in soils and soil size-fractions and their changes due to deforestation and pasture installation in eastern Amazon. Geoderma, v.61, p.103-118, 1994.

ELliot, E.T.; CAMBARDELlA, C.A. Physical separation of soil organic matter. Agricultural Ecosystem and Environment, v.34, p.407-419, 1991.

EMBRAPA. Serviço Nacional de Levantamento e Conservação de Solos (Rio de Janeiro, RJ). Manual de métodos de análises do solo. Rio de Janeiro : Ministério da Agricultura, 1979. não paginado.

FEIGL, B.J.; MELILLO, J.; CERRI, C.C. Changes in the origin and quality of soil organic matter after pasture introduction in Rondonia (Brazil). Plant and Soil, v.175, p.21-29, 1995.

FELLER, C. La matière organique dans les sols tropicaux à argile 1:1: recherche de compartiments organiques fonctionnels. Une approche granulométrique. Paris : ORSTOM, 1995. $393 p$ + annexes. (Collection ORSTOM TDM, 144).

FELLER, C. Organic inputs, soil organic matter and functional soil organic compartments in low activity clay soils in tropical zones. In: MULONGOY, K.; MERCKX, R. (Eds.). Soil organic matter dynamics and sustainability of tropical agriculture. New York : John Wiley, 1993. p.77-88.

FELLER, C. Une méthode de fractionnement granulométrique de la matière organique des sols. Cahier ORSTOM: Série Pédologie, v.17, p.339-346, 1979.

FELLER, C.; ALBRECHT, A.; TESSIER, D. Aggregation and organic matter storage in kaolinitic and smectitic tropical soils. In: CARTER, M.R.; STEWART, B.A. (Eds.). Structure and organic matter storage in agricultural soils. Boca Raton : CRC Lewish, 1996. Ch.8, p.309-352. (Advances in Soil Science).

Pesq. agropec. bras., Brasília, v.35, n.1, p.157-170, jan. 2000 
FELLER, C.; BURTIN, G.; GERARD, B.; BALESDENT, J. Utilisation des résines sodiques et des ultrasons dans le fractionnement granulométrique de la matière organique des sols. Intérêt et limites. Science du Sol, Paris, v.29, p.77-94, 1991a.

FELlER, C.; CASABIANCA, H.; CERRI, C.C. Renouvellement du carbone des fractions granulométriques d'un sol ferrallitique forestier (Brésil) mis en culture de canne à sucre. Étude par le ${ }^{13} \mathrm{C}$ en abondance naturelle. Cahier ORSTOM: Série Pédologie, Paris, v.26, p.365-369, 1991b.

FELLER, C.; FRANÇOIS, C.; VILLEMIN, G.; PORTAL, J.M.; TOUTAIN, F.; MOREL, J.L. Nature des matières organiques associées aux fractions argileuses d'un sol ferrallitique. Compte Rendu Académie des Sciences, Paris, tomo 312, Série II, p.1491-1497, 1991c.

FREITAS, P.L. de; BLANCANEAUX, Ph.; MOREAU, R. Caractérisation des sols des Cerrados Brésiliens (Savanes) dans le cadre de l'analyse morphostructurale. In: RÉUNION DU GROUPE THÉMATIQUE STRUCTURE ET FERTILITÉ DES SOLS TROPICAUX, 3., 1995, Montpellier. Compte Rendu. Montpellier : ORSTOM, 1996. p.6-23.

GAVINELLI, E.; FELLER, C.; LARRÉ-LARROUY, M.C.; BACYE, B.; DJEGUI, N.; NZILA, J. de D. A routine method to study soil organic matter by particle-size fractionation: Examples for tropical soil. Communications in Soil Science and Plant Analysis, v.26, p.1749-1760, 1995.

GOEDERT, W.J. Management of acid tropical soils in the savannas of South America. In: IBSRAM, INAUGURAL WORKSHOP, 1985, Yurimaguas, Peru. Proceedings. Bangkok : IBSRAM, 1987. p.109-127.

GOLCHIN, A.; OADES, J.M.; SKJEMSTAD, J.O.; CLARKE, P. Structural and dynamic properties of soil organic matter as reflected by $\mathrm{C}-13$ natural abundance, phyrolysis mass spectrometry and solid-state C-13 NMR spectroscopy in density fraction of an Oxisol under forest and pasture. Australian Journal of Soil Research, v.33, n.1, p.59-76, 1995.

KOUAKOUA, E. La matière organique et la stabilité structurale d'horizons de surface de sols argileux tropicaux. Effet du mode de gestion des sols. Nancy : Université Nancy I, 1997. 209p. Thèse de Doctorat.

LEPSCH, I.F.; MENK, J.R.F.; OLIVEIRA, J.B. de. Carbon storage and other properties of soils under agriculture and natural vegetation in São Paulo State, Brazil. Soil Use and Management, v.10, p.34-42, 1994.

MARTIN, A.; MARIOTTI, A.; BALESDENT, J.; LAVELLE, P.; VUATTOUX, R. Estimate of organic matter turnover rate in a savanna soil by ${ }^{13} \mathrm{C}$ natural abundance measurements. Soil Biology \& Biochemistry, v.22, p.517-523, 1990.

MOREAU, R. Évolution des sols sous différents modes de mise en culture en Côte d'Ivoire forestière et préforestière. Cahier ORSTOM: Série Pédologie, Paris, v.20, n.4, p.311-325, 1983.

NASCIMENTO, V.M.; ALMENDROS, G.; FERNANDES, F.M. Evolution patterns of the soil organic matter in some agricultural systems in the Brazilian "Cerrado" region. European Journal of Soil Biology, v.29, p.177-182, 1993.

PIERI, C. Fertilité des terres de savanes. Paris : Ministère de la Coopération - CIRAD, 1989. 444p.

RESCK, D.V.S.; PEREIRA, J.; SILVA, J.E. da. Dinâmica da matéria orgânica na região dos Cerrados. Planaltina, DF : Embrapa-CPAC, 1991. 21p. (Embrapa-CPAC. Documentos, 36).

TIESSEN, H.; STEWART, J.W.B. Light and electron microscopy of stained microaggregates: the role of organic matter and microbes in soil aggregation. B iogeochemistry, v.5, p.312-322, 1988. 